\title{
ELLERMAN BOMBS AT HIGH RESOLUTION. IV. VISIBILITY IN Na I AND Mg I
}

\author{
R. J. Rutten ${ }^{1,2,3}$, L. H. M. Rouppe VAn Der Voort ${ }^{1}$, AND G. J. M. VisSers ${ }^{1}$ \\ ${ }^{1}$ Institute of Theoretical Astrophysics, University of Oslo, P.O. Box 1029, Blindern, N-0315 Oslo, Norway \\ ${ }^{2}$ Lingezicht Astrophysics, 't Oosteneind 9, 4158 CA Deil, The Netherlands; R.J.Rutten@uu.nl \\ Received 2015 March 11; accepted 2015 May 11; published 2015 July 28
}

\begin{abstract}
Ellerman bombs are transient brightenings of the wings of the solar Balmer lines that mark reconnection in the photosphere. Ellerman noted in 1917 that he did not observe such brightenings in the $\mathrm{Na}$ I D and Mg I b lines. This non-visibility should constrain EB interpretation, but has not been addressed in published bomb modeling. We therefore test Ellerman's observation and confirm it using high-quality imaging spectrometry with the Swedish 1-m Solar Telescope. However, we find a diffuse brightness in these lines that seems to result from prior EBs. We tentatively suggest this is a post-bomb hot-cloud phenomenon also found in recent EB spectroscopy in the ultraviolet.
\end{abstract}

Key words: Sun: activity - Sun: atmosphere - Sun: magnetic fields

\section{INTRODUCTION}

Ellerman (1917) discovered intense short-lived brightenings of the extended wings of the Balmer $\mathrm{H} \alpha$ line at $6563 \AA$ in complex solar active regions which he called "solar hydrogen bombs." They have been called "Ellerman bombs" (henceforth abbreviated to EB) since McMath et al. (1960) but they are also called "moustaches" after Severny (1956; who did not call them bombs but did invoke nuclear explosions). For more detail we refer to our review of the extensive EB literature in Rutten et al. (2013).

In the preceding EB studies of this series we used highquality imaging spectroscopy and spectropolarimetry with the Swedish 1-m Solar Telescope (SST). Paper I (Watanabe et al. 2011) established that EBs are a purely photospheric phenomenon. Paper II (Vissers et al. 2013) added evidence that EBs mark magnetic reconnection of strong opposite-polarity field concentrations in the low photosphere and discussed their appearance in ultraviolet continuum images from the Atmospheric Imaging Assembly (AIA) of the Solar Dynamics Observatory (SDO). Paper III (Vissers et al. 2015) discussed the appearance of EBs in ultraviolet lines in spectra taken with the Interface Region Imaging Spectrograph (IRIS; De Pontieu et al. 2014).

In this installment we address the visibility of EBs in the $\mathrm{Na}$ I D and Mg I b lines. Ellerman (1917) already remarked that his bombs did not appear in these lines, nor in the continuum. We check his claims here exploiting the high resolution of the SST because both provide important formation constraints for EB interpretation.

The absence of EBs in the continuum was already an important constraint for the EB modeling by Kitai (1983), Berlicki et al. (2010), Bello González et al. (2013), and Berlicki \& Heinzel (2014) who all applied ad-hoc perturbations of a static standard model and NLTE line synthesis to reproduce observed $\mathrm{H} \alpha$ moustaches. We summarize these studies briefly.

Kitai (1983) fitted EB profiles in $\mathrm{H} \alpha$ and $\mathrm{Ca}$ II $\mathrm{H}$ and $\mathrm{K}$ spectrograms taken at Hida Observatory by perturbing the VAL3C standard model atmosphere of Vernazza et al. (1981). $\mathrm{He}$ concluded that temperature enhancement of $1500 \mathrm{~K}$ and

\footnotetext{
3 http://www.staff.science.uu.nl/rutte101.
}

density enhancement by a factor of 5 were needed at heights 700-1200 km, i.e., in the VAL3C chromosphere, and excluded deeper onsets because these predicted continuum brightening.

Berlicki et al. (2010) modeled EB contrasts in Lyot-filter $\mathrm{H} \alpha$ images from the Dutch Open Telescope (DOT) and simultaneous ultraviolet images at $1600 \AA$ from the TRACE satellite by perturbing a similar but more recent standard model, but only its temperature. They concluded that a humplike increase of about $3000 \mathrm{~K}$ in the upper photosphere would explain their measurements.

In a similar analysis Berlicki \& Heinzel (2014) used another DOT data set which also provided concurrent $\mathrm{Ca}$ II $\mathrm{H}$ images and extended the model perturbations to large grids, also modifying the density. Their best fit was for a model only perturbing the temperature, by $4000 \mathrm{~K}$ at height $1000 \mathrm{~km}$ in the onset of the model chromosphere.

The analysis by Bello González et al. (2013) was the most elaborate, observationally by using Fabry-Pérot imaging spectroscopy in $\mathrm{H} \alpha$ with concurrent full-Stokes polarimetry at the German Vacuum Tower Telescope, and in modeling by not applying the 1D plane-parallel layer assumption taken by the other authors but instead performing 2D $\mathrm{H} \alpha$ synthesis for imposed EB perturbations embedded within a standard model and including slanted limbward viewing. They obtained best fits from temperature increases in the $300-800 \mathrm{~km}$ height range up to $5000 \mathrm{~K}$ (doubling of their standard model) and simultaneous increase of the $\mathrm{H} \alpha$ opacity by a factor of five.

In all of these models, the proposed temperature enhancements were humps starting above $300 \mathrm{~km}$ or higher to avoid enhancements of the optical continuum. However, our highresolution observations in Paper I contradict such high EB onset because they show that actual EBs are rooted deep in strong-field magnetic concentrations in network lanes, without apparent gap of such size. This lower-part EB visibility in $\mathrm{H} \alpha$ remains unexplained.

A potential second failure of the models is that none was verified with respect to Ellerman's non-appearance of EBs in the $\mathrm{Mg}_{\mathrm{I}} \mathrm{b}$ and $\mathrm{Na}$ I D lines. In standard models these lines sample the heights of the imposed temperature enhancements (Rutten et al. 2011), so that the EB models will probably predict EB brightening in them. 
A third, more recent, issue is the pronounced visibility of EBs in the $\mathrm{C}_{\text {II }} 1334$ and $1335 \AA$ and Si IV 1394 and $1403 \AA$ doublets sampled by IRIS which we reported in Paper III. It seems unlikely that the published models can match their brightenings since they impose EB temperatures reaching $10,000 \mathrm{~K}$ at most, whereas the characteristic formation temperature of the $\mathrm{Si}$ Iv lines is $80,000 \mathrm{~K}$ (De Pontieu et al. 2014).

In this paper we concentrate on the earlier issues: the nonappearance of EBs in the continuum and in the NaID and Mg I b lines. We use high-resolution SST observations to confirm Ellerman's claims, but we find more diffuse brightenings that seem related to prior EB activity detected in SDO/ AIA images. These may represent hot EB aftermaths as those diagnosed from IRIS spectra (Paper III).

In Section 2 we describe the SST observations taken for this analysis and in Section 3 the results, including comparison with SDO images. The conclusions follow in Section 4.

\section{OBSERVATIONS}

2013 July 4 was a day of variable seeing at the SST (Scharmer et al. 2003) on La Palma. These conditions and the presence of an emerging active region toward the limb were suited to test Ellerman's claims through imaging spectroscopy with the CRisp Imaging SpectroPolarimeter (CRISP; Scharmer et al. 2008), a profile-scanning Fabry-Pérot interferometer. The target was AR 1785 at solar $(X, Y)=(-693,-189)$ arcsec from the disk center with viewing angle $\mu=0.65(\theta=49.5)$.

Dual profile scanning of $\mathrm{H} \alpha$ and the $\mathrm{Na}_{\mathrm{ID}} \mathrm{D}_{1}$ line at 5895.94 A was performed during 11:09-11:26 UT, followed by dual profile scanning of $\mathrm{H} \alpha$ and the $\mathrm{Mg}_{\mathrm{I}} \mathrm{b}_{2}$ line at 5172.70 A during 11:28-11:41 UT (wavelengths for standard air from Moore et al. 1966). The scan cadence was $20 \mathrm{~s}$.

$\mathrm{H} \alpha$ was sampled at 39 wavelengths over $\Delta \lambda= \pm 2.06 \AA$ from the center of the profile after averaging over the full field of observation with equidistant spacing $\Delta \lambda=0.086 \AA$ across its core and wider spacing in its wings. $\mathrm{Na}_{\mathrm{I}} \mathrm{D}_{1}$ was sampled at 41 wavelengths over $\Delta \lambda= \pm 1.71 \AA$ with a narrowest core sampling of $\Delta \lambda=0.028 \AA ; \mathrm{Mg} \mathrm{I}_{2}$ was similarly sampled over $\Delta \lambda= \pm 0.89 \AA$.

Movies based on these data showed that the EBs present in $\mathrm{H} \alpha$ were not evident in $\mathrm{Na}_{\mathrm{I}} \mathrm{D}_{1}$ or $\mathrm{Mg} \mathrm{I}_{2}$, but they did suggest that at the locations where $\mathrm{H} \alpha \mathrm{EBs}$ went off, diffuse brightening in these lines subsequently occurred.

Because the image quality was too variable for time sequence analysis, we selected the sharpest moment of each dual profile scan and performed full reduction only for these, using procedures described by de la Cruz Rodríguez et al. (2015). These include dark- and flat-field correction; multiobject, multi-frame blind deconvolution following van Noort et al. (2005) to reduce the effects of high-order atmospheric seeing not already corrected by the adaptive optics of the SST; removal of the remaining small-scale distortions between the different line samplings; and correction for the prefilter transmission profile.

We also collected corresponding longer-duration image sequences from SDO/AIA (Lemen et al. 2012) and SDO/ HMI (Scherrer et al. 2012) using the JSOC image cutout service at Stanford University. They were co-aligned with the SST images using IDL programs available on the website of the first author.
In the alignment and the data analysis, we made much use of the CRisp SPectral EXplorer (CRISPEX; Vissers \& Rouppe van der Voort 2012) for data browsing.

\section{RESULTS}

Overview-Figure 1 provides an overview of the observed area at the time of the best SST H $\alpha-\mathrm{Na}_{\text {I }} \mathrm{D}_{1}$ scan.

The SDO/HMI magnetogram in the first panel illustrates the magnetic complexity of the emerging region with many adjacent opposite-polarity field patches. The next two panels sample the blue and red outer wings of $\mathrm{H} \alpha$ and contain multiple EBs. They tend to be located near adjacent opposite-polarity field patches in the first panel. However, the sensitivity and resolution of HMI are too poor to resolve the cancelation of small opposite-polarity patches that characteristically occurs at EB sites (Paper II).

The fourth panel illustrates that EBs take place well below the thick fibrilar canopy that the $\mathrm{H} \alpha$ line core invariably displays in such crowded active regions. The bright feature just left of image center is not an EB.

The AIA $1700 \AA$ image in the bottom row illustrates that EBs are well observable in this diagnostic (Paper II). The round, bright features show good correspondence with the $\mathrm{H} \alpha \mathrm{EBs}$, even at inferior spatial resolution. In Paper II, we showed that the morphology is not identical; in Paper III we showed that in slanted viewing, the $1700 \AA$ images show mostly the downward-moving, cooler lower part of an EB, whereas the ultraviolet IRIS diagnostics favor the upward-moving, hotter upper part. The whole EB is seen in the $\mathrm{H} \alpha$ wings.

In the AIA $1600 \AA$ image in the second row of Figure 1, the EBs reach yet larger brightness contrast, but here the non-EB feature left of center in the $\mathrm{H} \alpha$ core image also appears to be very bright. This is a small flaring arch filament (FAF), the brightness of which is likely contributed by the $\mathrm{C}$ IV doublet at 1548 and $1550 \AA$ in this passband. Such small FAFs typically have elongated shapes, as is the case here, live shorter lifetimes, show rapid apparent motion along filamentary strands, and also stand out in higher-temperature AIA diagnostics (Paper III). This feature is also prominent in the remaining two panels showing AIA's 304 and $171 \AA$ images, whereas the EBs leave no signature in these (Paper II).

In passing, we note that the darkest $\mathrm{H} \alpha$ line-core fibrils are also visible in the hot AIA diagnostics. The darkest fibrils have the largest neutral-hydrogen density (Leenaarts et al. 2012), and hence block short-wavelength radiation by incoherent bound-free scattering out of the narrow AIA passbands (Rutten 1999).

$E B s$ in $N a_{\text {I }} D_{1}$ and $M g$ I $b_{2}$-Figure 2 presents some EBs in more detail, including the scene in $\mathrm{Na}_{\mathrm{I}} \mathrm{D}_{1}$ and $\mathrm{Mg} \mathrm{b}_{2}$ for the subfield defined by the frame in Figure 1.

The first column shows this area in the blue wing of $\mathrm{H} \alpha$. In limbward viewing at this image quality, the distinction between EBs and much more ubiquitous quiescent magnetic concentrations is easily made. However, these have often been confused, both in the older EB literature (see Rutten et al. 2013) and also more recently (details in Paper III).

Although the rows differ by nearly 8 minutes, a pair of unmistakable and rather similar EBs is seen below $\mathrm{A}$ in the two first-column images. We would not call this the same EB because EBs tend to come in rapid succession, appearing as "flickering flames" whose feet travel along an intergranular lane filled with magnetic concentrations (Paper I) canceling 

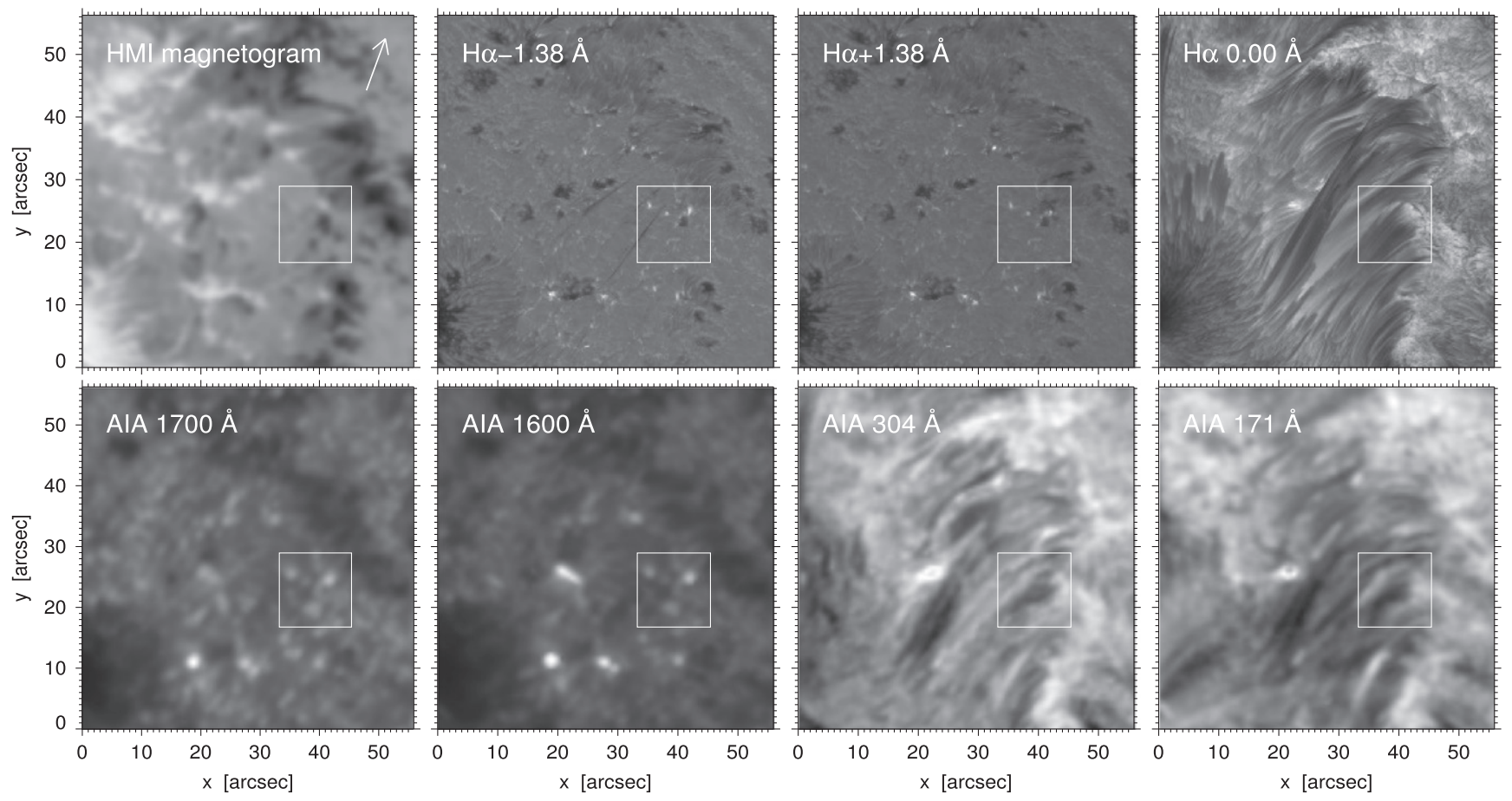

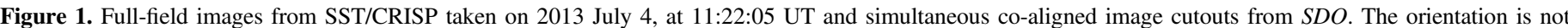

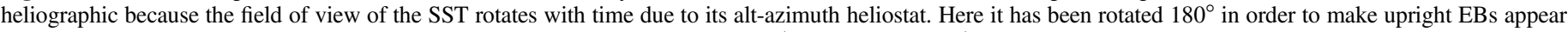

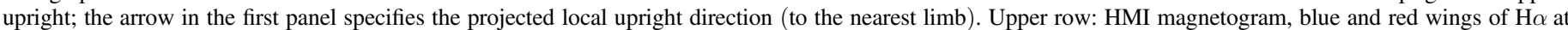

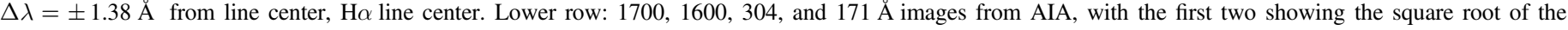
measured intensity and the other two the logarithm. The white frame outlines the small cutout subfield of Figures 2 and 7.
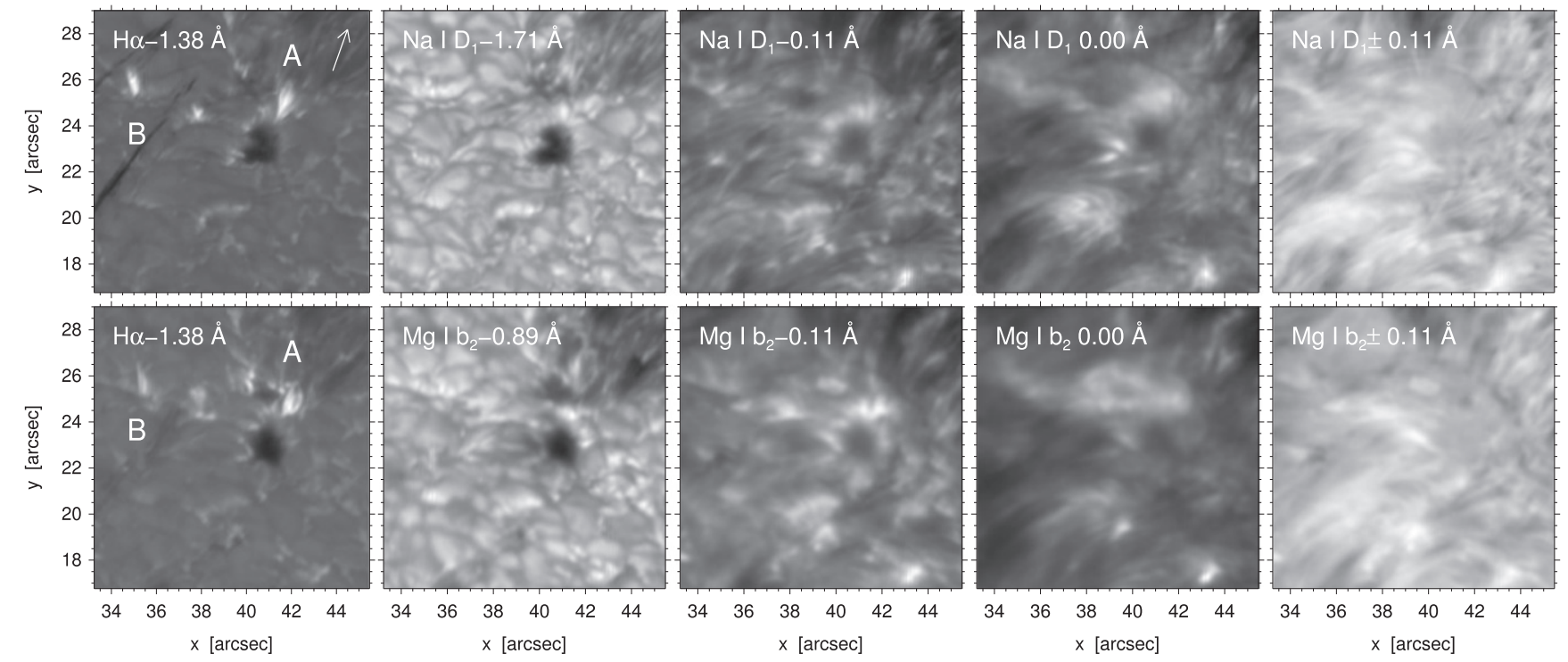

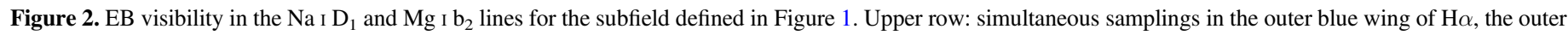

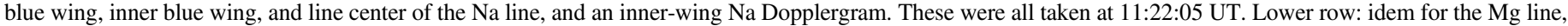

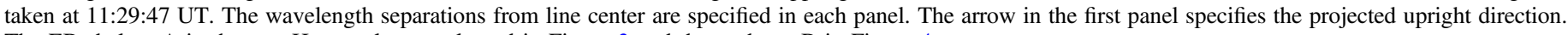
The EBs below A in the two H $\alpha$ panels are enlarged in Figure 3 and those above B in Figure 4.

against incoming fresh opposite-polarity flux (Paper II). This often happens repetitively in sequences which may last many minutes and may even repeat over an hour or longer (Paper III). In this case, at eight minutes of separation, this pair shows a small shift in location but roughly similar morphology, including upward splits which suggest reconnection along different field lines or multiple field-guided jets. Both show upright orientation at least in their azimuthal projection.
Smaller EBs are seen above B in both $\mathrm{H} \alpha$ samples, and also at similar locations along field-filled lanes in the two panels.

The second column shows outer-wing $\mathrm{Na}_{\mathrm{I}} \mathrm{D}_{1}$ and $\mathrm{Mg}_{\mathrm{I}} \mathrm{b}_{2}$ images of the same scene at the two sample times. Each is the starting wavelength of the CRISP scan. For $\mathrm{Na}_{\mathrm{I}} \mathrm{D}_{1}$ this is in the continuum. For $\mathrm{Mg}_{\mathrm{I}} \mathrm{b}_{2}$, which has extended wings wider than the CRISP prefilter passband and also deep blends, it is already at $26 \%$ of the line depth. 

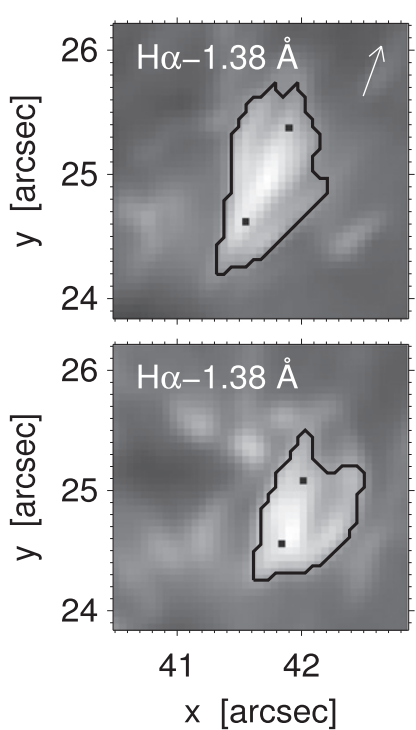
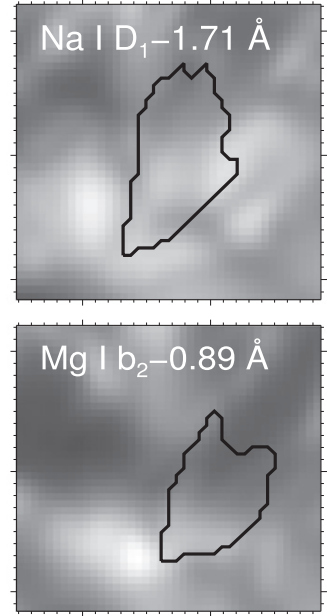

4142

$x$ [arcsec]
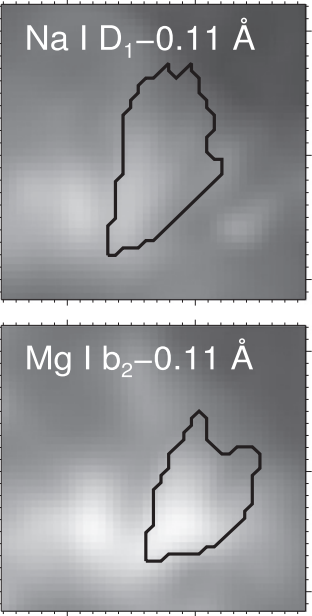

$41 \quad 42$

$x$ [arcsec]
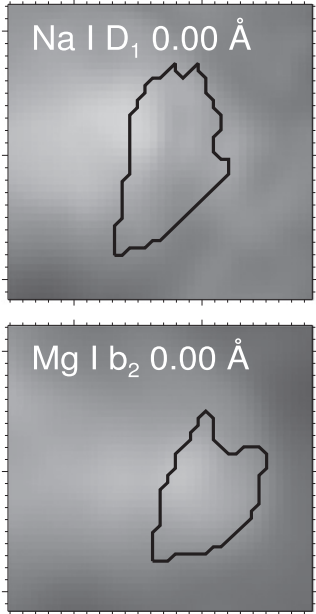

$41 \quad 42$

$x$ [arcsec]
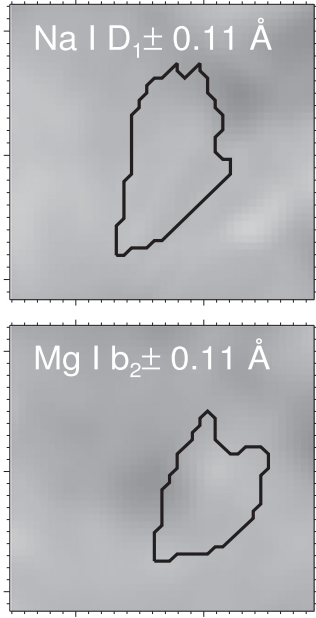

$41 \quad 42$

$x$ [arcsec]

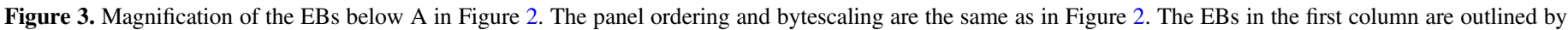

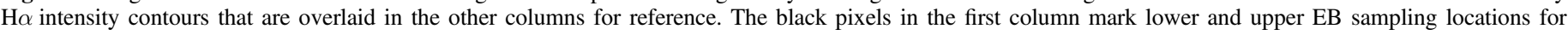
Figure 5.

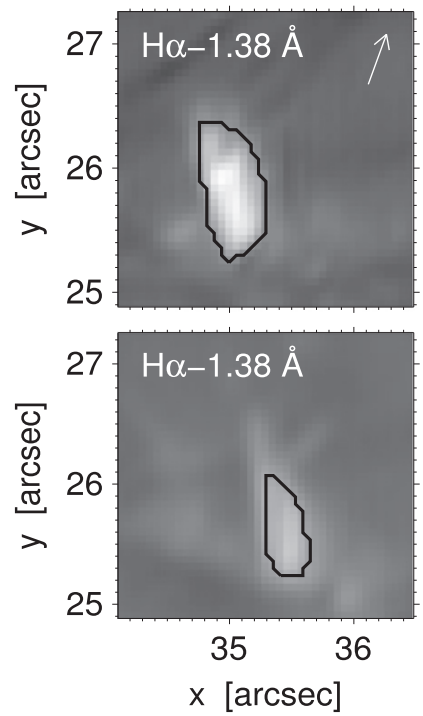

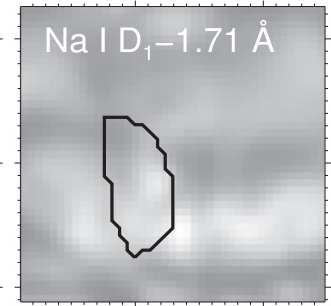
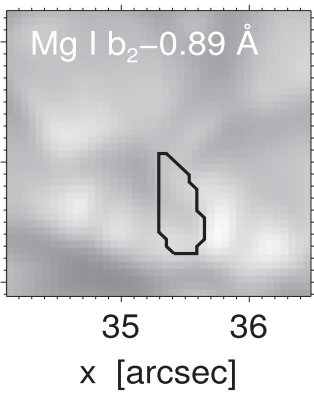
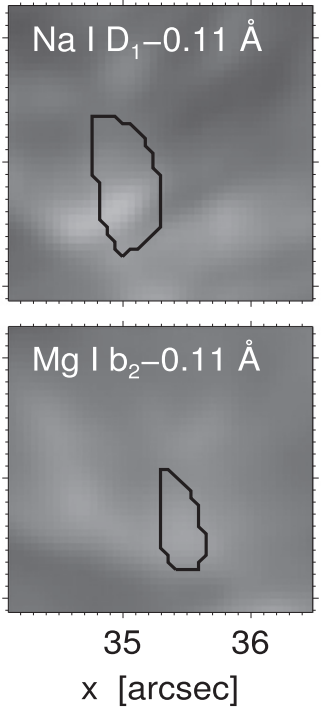
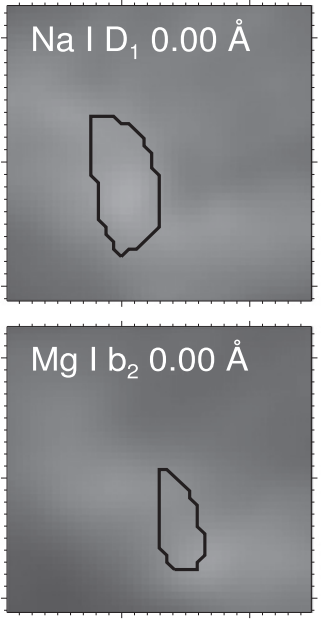

$$
\begin{gathered}
35 \quad 36 \\
\times \text { [arcsec] }
\end{gathered}
$$
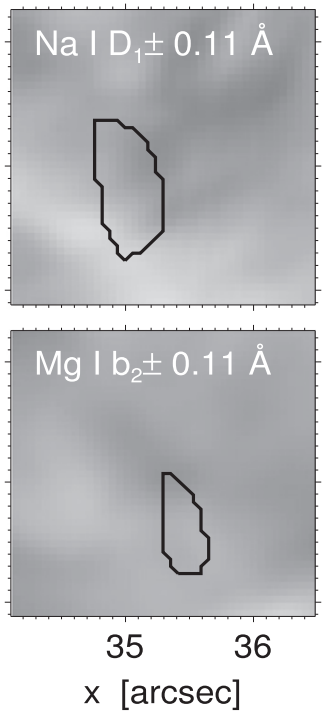

Figure 4. Magnification of the EBs above B in Figure 2 in the format of Figure 3.

The sharpness of the granulation and of numerous intergranular magnetic bright points in the second panel illustrates the superior image quality of the SST. The EBs in the first panel are transparent in the second (shown at larger magnification in Figures 3 and 4), in agreement with Ellerman's statement that EBs do not brighten the continuum. The granulation scene in the lower panel of the second column differs not only from pattern evolution during the eight minute sampling interval, but also from different sampling through higher line-wing formation (detailed in Rutten et al. 2011).

A comparison with the $\mathrm{H} \alpha$ blue-wing granulation in the first column illustrates the substantial "flattening" of granulation in the latter diagnostic (Leenaarts et al. 2005), which contributes to the relatively high contrast of intergranular magnetic concentrations in the blue $\mathrm{H} \alpha$ wing (Leenaarts et al. 2006), although they remain less bright than EBs (Paper I and Paper II).
The third and fourth columns sample the $\mathrm{Na}_{\mathrm{I}} \mathrm{D}_{1}$ and $\mathrm{Mg}_{\mathrm{I}} \mathrm{b}_{2}$ lines in their inner blue wings and at their centers. These images are less sharp than those of far wing (also of $\mathrm{H} \alpha$ ) because the latter are formed in LTE whereas the former belong to the profile part where scattering dominates heavily in each line, particularly at its center (Rutten et al. 2011).

Because some contrast features in the inner wings are likely not caused by profile raising or lowering but by core Dopplershift, we add Dopplergrams at this sampling wavelength in the final column. They are defined as blue-wing intensity minus red-wing intensity normalized by their sum; the redshift of the absorption core then produces enhanced brightness and also brightens line-center samples.

The EBs do not stand out in these Dopplergrams, but other small bright inner-wing features do. An example is the bright feature at $(x, y)=(43,17)$ in both rows. It is not an EB, but a core-redshift, a core plus blue-wing emission feature, or both 

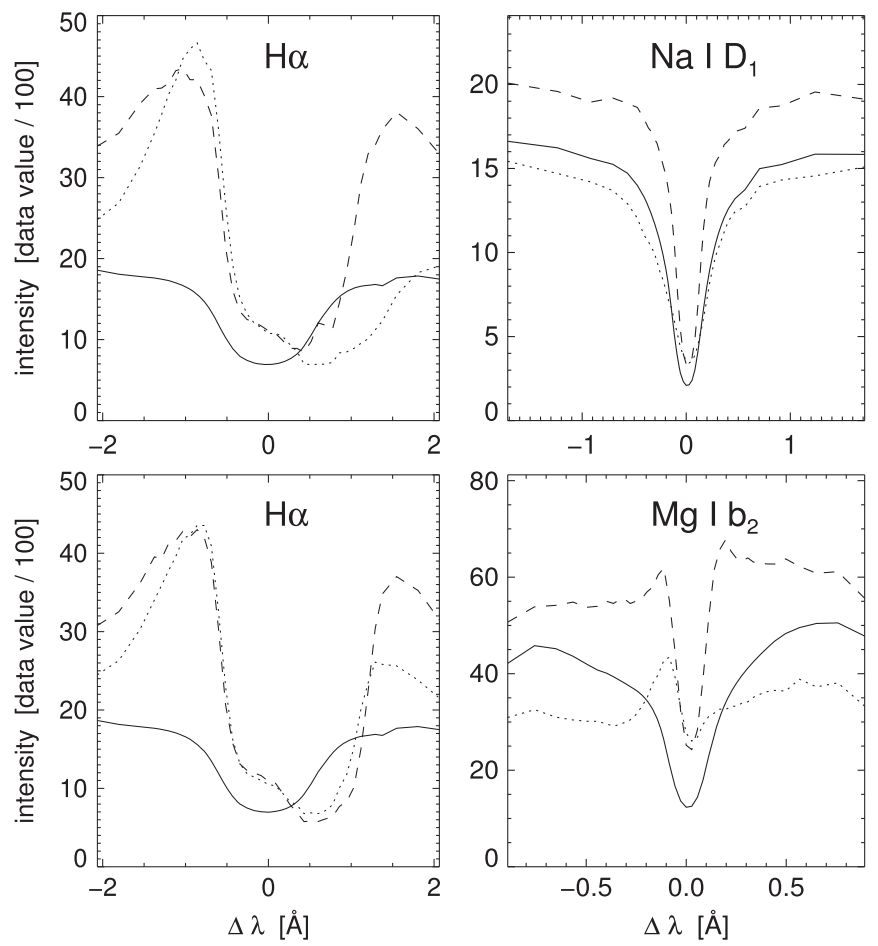

Figure 5. Spectral profiles at the two sampling times. Solid: mean over the whole field of view. Dashed: lower EB pixel specified in the first column of Figure 3. Dotted: upper pixel. The wavelengths are relative to the line center of the mean profiles. The intensities are data readouts divided by 100 .

acting together as the shock grain in the first quartet of Figure 9 of Leenaarts et al. (2010).

These various samples of $\mathrm{NaI}_{1} \mathrm{D}_{1}$ (upper row) and $\mathrm{Mg} \mathrm{I}_{2}$ (lower row) represent our high-resolution test of Ellerman's statement that his bombs do not show up in these lines. Indeed, although there are small bright features in these panels, there is obviously no direct $1: 1$ correspondence with the $\mathrm{H} \alpha$ EBs at left. None of the latter would be identified as an EB from the other images. The closest is the bright EB pair below A, which seems to show at least some corresponding brightness in the two $-0.11 \AA$ panels without Doppler signature.

In Figures 3 and 5 we examine the EB pair below A in detail. Figure 3 magnifies them in the format of Figure 2. The second panel confirms that the first EB is transparent in the continuum. The third column does indeed suggest some morphological similarity between the lower part of the $\mathrm{H} \alpha \mathrm{EB}$ and the slight brightness enhancements in the $-0.11 \AA$ samplings, especially in $\mathrm{Mg}_{\mathrm{I}} \mathrm{b}_{2}$ for the second $\mathrm{EB}$, but weaker than in the $\mathrm{H} \alpha$-wing. Other nearby features are as bright.

The line centers (fourth column) show no clear signature of the upper parts of the EBs, although their height is $1 \mathrm{Mm}$ or more (projected lengths in the first column) so that their top reaches higher than the normal formation height of the $\mathrm{Na}_{\mathrm{I}} \mathrm{D}_{1}$ and $\mathrm{Mg}_{\mathrm{I}} \mathrm{b}_{2}$ cores (Leenaarts et al. 2010; Rutten et al. 2011). The lower parts of EBs may be shielded by adjacent undisturbed gas producing normal line cores in slanted viewing, but their tops should jut out beyond this layer and be visible if enhanced in these lines. This is also evident in the IRIS spectra in Paper III, in which slanted view lines toward lower EB parts show deep $\mathrm{Mn}$ I blends on $\mathrm{Mg}$ II $\mathrm{h}$ and $\mathrm{k}$ from adjacent undisturbed upper-photosphere gas while these blends vanish in the upper spectra. The conclusion is that the lower parts of these two EBs may show brightening in $\mathrm{Na}_{I_{1}} \mathrm{D}_{1}$ and especially $\mathrm{Mg}_{\mathrm{I}} \mathrm{b}_{2}$, but that the upper parts do not.
The final column shows no pronounced Dopplershifts, whereas in sampling an EB one expects redshift (dark for a bright feature) for the lower part and blueshift for the upper part (Paper III).

Figure 5 completes our detailed examination of these two EBs by showing line profiles for the lower and upper samplings marked by black pixels in Figure 3. They confirm that only the lower EB parts give noticeable wing enhancements in $\mathrm{Na}_{I} \mathrm{D}_{1}$ and $\mathrm{Mg}_{\mathrm{I}} \mathrm{b}_{2}$, but that these remain smaller than for $\mathrm{H} \alpha$. The overlying fibril that redshifted the $\mathrm{H} \alpha$ core did not redshift the other line cores, but one might speculate that the single blue-side peak in $\mathrm{Mg}_{\mathrm{I}} \mathrm{b}_{2}$ from the upper part testifies to an upward EB jet (dotted profile).

We have examined all other EBs in Figure 1 in comparable detail, by using CRISPEX to inspect and blink our various diagnostics at such high magnification, but found no other with better correspondence that the pair detailed above. Generally they exhibit brightening in the inner wings of $\mathrm{Mg} \mathrm{I}_{2}$, but not more than in many other features due to network, reversed granulation, or shocks (see Rutten et al. 2011). Na I $D_{1}$ shows less EB brightening or none. The $\mathrm{Mg}_{\mathrm{I}} \mathrm{b}_{2}$ profiles only rarely display core asymmetry as for the upper pixel in Figure 5. Thus, while Figures 3 and 5 suggest some EB signature, especially in $\mathrm{Mg}_{\mathrm{I}} \mathrm{b}_{2}$, this is an exceptional case and only a poor case at that.

We selected the EBs below A in Figure 2 and detailed these in Figures 3 and 5 as representing a best effort in trying to counter Ellerman's claim. Its selection as such is illustrated by Figure 4, which repeats the magnified format of Figure 3 for the EBs above B in Figure 2. In both $\mathrm{H} \alpha$ images, these are also unmistakable EB flames but they leave no signature in the other panels of Figure 4.

Furthermore, we demonstrate the selection of the EBs in Figure 3 as the best counter-example with the full-field scatter diagrams in Figure 6. The vertical line is one of the criteria defined in Paper II for automated EB detection. The mountain ridges to the left of these thresholds portray fairly good correlation between the granulation and network scenes, whereas the relatively few EB pixels lie to their right. In the upper panel, these show no correlation between high $\mathrm{H} \alpha$-wing intensity and high $\mathrm{NaI}_{\mathrm{I}} \mathrm{D}_{1}$-wing intensity, but in the lower panel the red pixels do show such bright-bright correlation for $\mathrm{Mg}_{\mathrm{I}} \mathrm{b}_{2}$. These appear within the contour in the lower panels of Figure 3.

Thus, also in this statistical measure, our selected EB had significant concurrent brightening in $\mathrm{Mg}_{\mathrm{I}} \mathrm{b}_{2}$, but this was indeed exceptional. The blue pixels for the contours in Figure 4 show no such correlation.

The upshot is that we confirm Ellerman's claim-nearly a century later.

Hot EB aftermaths-When we viewed our data as movies notwithstanding the bad-seeing moments, we noted a general presence of diffuse bright clouds in the line-center $\mathrm{Na}_{\text {I }} \mathrm{D}_{1}$ and $\mathrm{Mg}_{\mathrm{I}} \mathrm{b}_{2}$ sequences near locations where $\mathrm{H} \alpha$ EBs went off. Our impression was that such areas are subsequently marked by diffuse line-center brightness.

In the fourth column of Figure 2, such features are present above the pore and roughly mimic the arch spanned by the actual EBs in the $\mathrm{H} \alpha$ panels (first column). If one compares the latter with all other panels and asks oneself whether EBs leave some signature in these two lines, then these diffuse line-center clouds seem the most viable candidate. However, other diffuse 

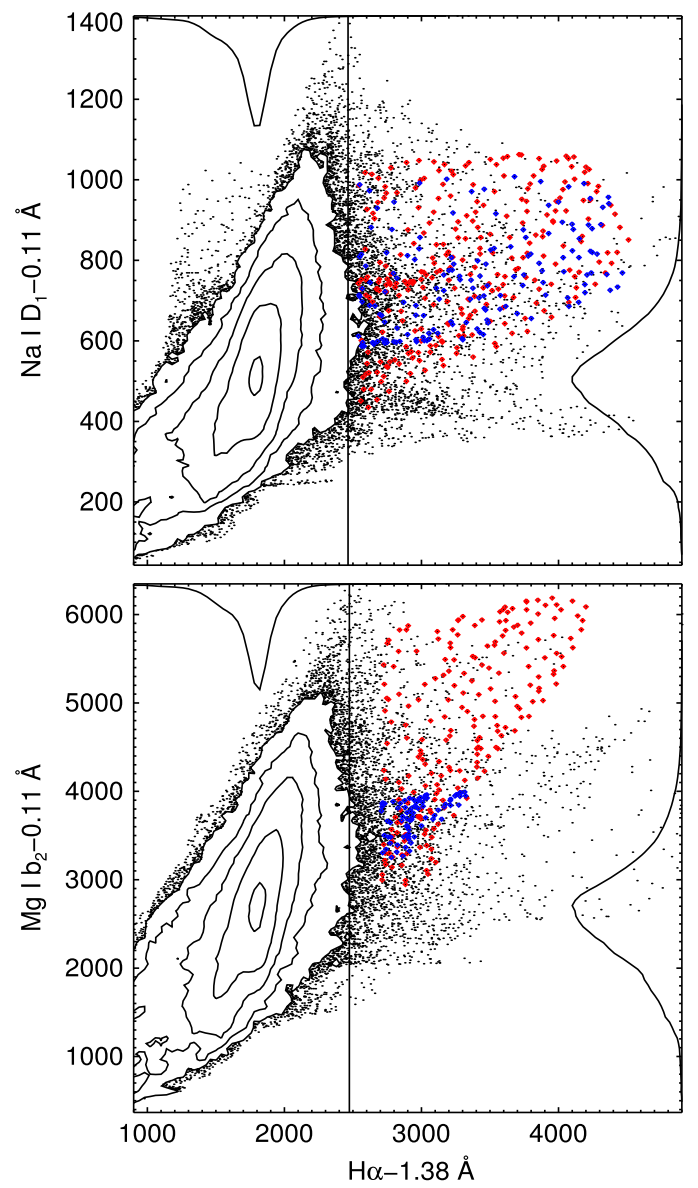

Figure 6. Scatter diagrams of $\mathrm{Na}$ I $\mathrm{D}_{1}$ (upper panel) and $\mathrm{Mg}$ I $\mathrm{b}_{2}$ (lower panel) wing intensities against $\mathrm{H} \alpha$ wing intensity for the full field of view (Figure 1). To avoid plot saturation, logarithmic-spaced sample density contours are plotted instead of individual pixel samples at large density. The histograms along the axes show normalized intensity distributions. The vertical lines mark $140 \%$ of the mean $\mathrm{H} \alpha$ value. Red pixels: within the EB contours in Figure 3. Blue pixels: within the EB contours in Figure 4.

brightness patches lower in the panels of Figure 2 do not correspond to EBs.

In Paper III, we found that during the aftermath of regular $\mathrm{H} \alpha$ EBs, there may appear features with highly enhanced emission in and very wide profiles of the ultraviolet Si IV and $\mathrm{C}$ II lines sampled by IRIS, and that these preferentially appear at sites with prolonged repeated EB activity.

In view of this parallel evidence for EB aftermaths, we tentatively suggest that prior EB activity may have caused diffuse brightness in $\mathrm{Na}_{\mathrm{I}} \mathrm{D}_{1}$ and $\mathrm{Mg}_{\mathrm{I}} \mathrm{b}_{2}$, and therefore turn to the $S D O$ image sequences in order to inspect the evolution prior to our SST snapshots. This is feasible because AIA 1700 and $1600 \AA$ images also display EBs, at least the stronger ones (Paper II and Figure 1). Although they do so less sharply than CRISP in $\mathrm{H} \alpha$ when there is good seeing at La Palma, they do so everywhere on the solar disk, all the time (24/7), at good cadence $(24 \mathrm{~s})$, and are readily available in the splendid SDO community service.

Figure 7 shows a time sequence of cutouts of HMI magnetograms and AIA 1700 and $1600 \AA$ images for the subfield defined in Figure 1 and shown in Figure 2. It shows that the bright EB in Figure 2, or rather its location, often also harbored a very bright EB before the SST samplings. Inspection of the earlier AIA data showed that this EB flaring
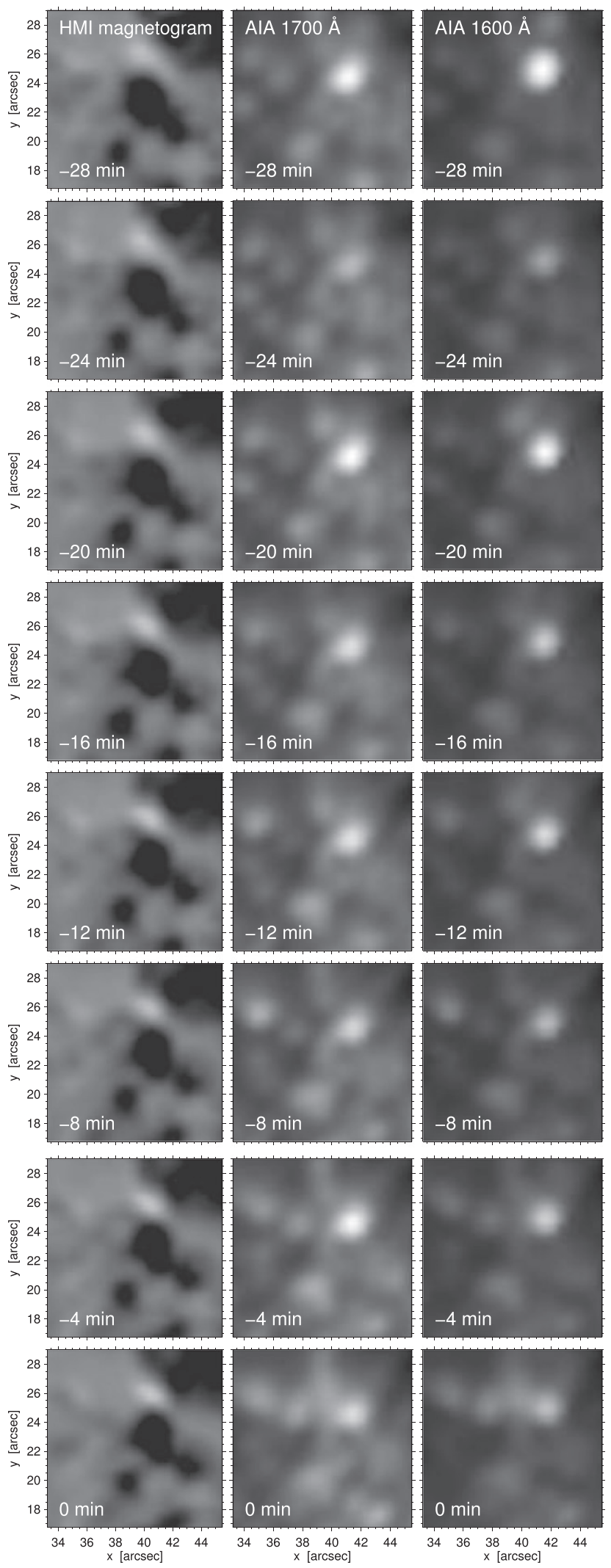

Figure 7. Time evolution prior to the image samples in Figures 1-3, and 5. Left to right: HMI magnetograms, AIA 1700, and $1600 \AA$ images. For each, the square root of the signal is shown. In addition, the magnetograms were clipped, which only affects the black polarity. The bytescaling remains the same along columns. The bottom row is for 11:29:45 UT, a few seconds after the lower row samplings in Figures 2, 3, and 5. The other rows span the prior evolution at 4 minute intervals; $\Delta t=-8$ minutes corresponds to the sampling time of Figure 1 and the upper rows of Figures 2, 3, and 5. 
actually started already an hour before and that it peaked at $\Delta t=-40$ minutes.

In particular, Figure 7 demonstrates that there were also bright EBs at this location during the dozen minutes preceding our first SST sampling and between our two samplings. The weaker EBs to the left of the brightest one in the $\mathrm{H} \alpha$ panels of Figure 2 were also present at $\Delta t=-4$ minutes and may have contributed to the arc-shaped bright cloud in the $\mathrm{Mg}_{\mathrm{I}} \mathrm{b}_{2}$ linecenter panel of Figure 2. This prior activity suggests that the diffuse cloud at the centers of $\mathrm{Na}_{\mathrm{I}} \mathrm{D}_{1}$ and $\mathrm{Mg}$ I $\mathrm{b}_{2}$ in Figure 2 may represent hot EB aftermaths analogous to those found in the IRIS spectra in Paper III.

We added the $1600 \AA$ column to Figure 7 in order to exclude FAFs as potential cause of such diffuse clouds because FAFs tend to have more dramatic hot aftermaths than EBs (Paper III). However, the scenes in 1700 and $1600 \AA$ in Figure 7 are nearly identical, apart from the larger EB contrast at $1600 \AA$ (darkening the background in our column-wise bytescaling). The prior brightenings also maintained characteristic roundish EB appearance and stability. Thus, there were no FAFs in this area during this period, and so the diffuse clouds were not an FAF product.

The sequence of HMI magnetograms in the first column suggests that the major patch of white polarity diminished on its lower right side where it was adjacent to the larger and stronger black patch and where the brightest EBs occurred. There were weaker-field encounters at the weaker EB sites. The resolution and sensitivity are insufficient to establish smallscale field cancelation, but these patterns suggest that it may well have happened.

\section{CONCLUSIONS}

Figure 2 confirms Ellerman's statement that his bombs do not brighten the $\mathrm{NaID}$ and $\mathrm{Mg}$ I b lines. Figures 3 and 5 suggest EB-foot brightening in their wings, but not much relative to other nearby features. Also, this is the best case; the other EBs in our samples show less correspondence.

However, at the line centers, there are diffuse bright clouds that may represent EB aftermaths comparable to those diagnosed from IRIS spectra in Paper III.

The interpretative suggestion from these results and from our results in Paper III is obvious: the upper parts of EBs are too hot to radiate in these lines, likely from neutral-species ionization, and hot enough to radiate in Si Iv lines. Much higher temperatures seem to be involved than in all EB modeling so far.

In addition, we speculate that in EB aftermaths, the neutral species may reappear through recombination in cooling postbomb "mushroom" clouds. For these, the observational suggestion is also obvious: perform similar dual-line imaging spectroscopy as presented here but a over longer duration and together with IRIS spectrometry, emphasizing EB and FAF aftermaths.
The challenge for EB modeling is to meet the wide set of diverse constraints posed by our high-resolution observations in $\mathrm{H} \alpha$ (Paper I and Paper II), the ultraviolet IRIS lines (Paper III), and the $\mathrm{Na}_{\mathrm{I}} \mathrm{D}_{1}$ and $\mathrm{Mg}_{\mathrm{I}} \mathrm{b}_{2}$ lines presented here. Modeling that explains all of these may also give insight into the nature of aftermath clouds.

This research was supported by the Research Council of Norway and by the European Research Council under the European Union's Seventh Framework Programme (FP7/20072013)/ ERC grant agreement No. 291058. The SST is operated by the Institute for Solar Physics of Stockholm University in the Spanish Observatorio del Roque de los Muchachos of the Instituto de Astrofísica de Canarias on the island of La Palma.

\section{REFERENCES}

Bello González, N., Danilovic, S., \& Kneer, F. 2013, A\&A, 557, A102 Berlicki, A., \& Heinzel, P. 2014, A\&A, 567, A110

Berlicki, A., Heinzel, P., \& Avrett, E. H. 2010, MmSAI, 81, 646

de la Cruz Rodríguez, J., Löfdahl, M. G., Sütterlin, P., Hillberg, T., \& Rouppe van der Voort, L. 2015, A\&A, 573, A40

de Pontieu, B., Title, A. M., Lemen, J. R., et al. 2014, SoPh, 289, 2733

Ellerman, F. 1917, ApJ, 46, 298

Kitai, R. 1983, SoPh, 87, 135

Leenaarts, J., Carlsson, M., \& Rouppe van der Voort, L. 2012, ApJ, 749, 136

Leenaarts, J., Rutten, R. J., Reardon, K., Carlsson, M., \& Hansteen, V. 2010, ApJ, 709, 1362

Leenaarts, J., Rutten, R. J., Sütterlin, P., Carlsson, M., \& Uitenbroek, H. 2006, A\&A, 449, 1209

Leenaarts, J., Sütterlin, P., Rutten, R. J., Carlsson, M., \& Uitenbroek, H. 2005 in ESA Special Publication 596, Chromospheric and Coronal Magnetic Fields, ed. D. E. Innes, A. Lagg \& S. A. Solanki (Les Ulis: EDP Sciences), 15

Lemen, J. R., Title, A. M., Akin, D. J., et al. 2012, SoPh, 275, 17

McMath, R. R., Mohler, O. C., \& Dodson, H. W. 1960, PNAS, 46, 165

Moore, C. E., Minnaert, M. G. J., \& Houtgast, J. 1966, The solar spectrum $2935 \AA$ to $8770 \AA$ (Washington, DC: USGPO)

Rutten, R. J. 1999, in ASP Conf. Ser. 184, Third Advances in Solar Physics Euroconference: Magnetic Fields and Oscillations, ed. B. Schmieder, A. Hofmann \& J. Staude (San Francisco, CA: ASP), 181

Rutten, R. J., Leenaarts, J., Rouppe van der Voort, L. H. M., et al. 2011, A\&A, 531, A17

Rutten, R. J., Vissers, G. J. M., Rouppe van der Voort, L. H. M., Sütterlin, P., \& Vitas, N. 2013, JPhCS, 440, 012007

Scharmer, G. B., Bjelksjo, K., Korhonen, T. K., Lindberg, B., \& Petterson, B. 2003, Proc. SPIE, 4853, 341

Scharmer, G. B., Narayan, G., Hillberg, T., et al. 2008, ApJL, 689, L69

Scherrer, P. H., Schou, J., Bush, R. I., et al. 2012, SoPh, 275, 207

Severny, A. B. 1956, Obs, 76, 241

van Noort, M., Rouppe van der Voort, L., \& Löfdahl, M. G. 2005, SoPh, 228, 191

Vernazza, J. E., Avrett, E. H., \& Loeser, R. 1981, ApJS, 45, 635

Vissers, G., \& Rouppe van der Voort, L. 2012, ApJ, 750, 22

Vissers, G. J. M., Rouppe van der Voort, L. H. M., \& Rutten, R. J. 2013, ApJ, 774, 32 (Paper II)

Vissers, G. J. M., Rouppe van der Voort, L. H. M., Rutten, R. J., Carlsson, M., \& de Pontieu, B. 2015, submitted (Paper III)

Watanabe, H., Vissers, G., Kitai, R., Rouppe van der Voort, L., \& Rutten, R. J. 2011, ApJ, 736, 71 (Paper I) 